Research Article

\title{
Analytical Properties for the Fifth Order Camassa-Holm (FOCH) Model
}

\author{
Mingxuan $\mathrm{Zhu}^{1}$, $\mathrm{Lu} \mathrm{Cao}^{2}$, Zaihong Jiang, (1), Zhijun Qiao ${ }^{3,}$, (1) \\ ${ }^{1}$ School of Mathematical Sciences, Qufu Normal University, Qufu 273100, P. R. China \\ ${ }^{2}$ Department of Mathematics, Zhejiang Normal University, Jinhua, 321004, P. R. China \\ ${ }^{3}$ School of Mathematical and Statistical Science, University of Texas Rio Grande Valley, Edinburg, TX 78539, USA
}

\section{ARTICLE INFO}

\section{Article History}

Received 21 March 2021

Accepted 06 May 2021

\section{Keywords}

The Fifth order Camassa-Holm (FOCH) model

global existence

infinite propagation speed

long time behavior

\section{Mathematics Subject}

Classifications

37L05

35Q35

35B44

\section{ABSTRACT}

This paper devotes to present analysis work on the fifth order Camassa-Holm (FOCH) model which recently proposed by Liu and Qiao. Firstly, we establish the local and global existence of the solution to the FOCH model. Secondly, we study the property of the infinite propagation speed. Finally, we discuss the long time behavior of the support of momentum density with a compactly supported initial data.

(c) 2021 The Authors. Published by Atlantis Press B.V. This is an open access article distributed under the CC BY-NC 4.0 license (http://creativecommons.org/licenses/by-nc/4.0/).

\section{INTRODUCTION}

In this paper, we consider the following fifth order Camassa-Holm (FOCH) model [29]:

$$
\left\{\begin{array}{l}
m_{t}+u m_{x}+b u_{x} m=0, \quad t>0, x \in \mathbb{R}, \\
m=\left(1-\alpha^{2} \partial_{x}^{2}\right)\left(1-\beta^{2} \partial_{x}^{2}\right) u, t>0, x \in \mathbb{R},
\end{array}\right.
$$

where $b \in \mathbb{R}$ is a constant, $\alpha, \beta \in \mathbb{R}$ are two parameters, $\alpha \neq \beta, \alpha \beta \neq 0$. Without loss of generality, we only consider the case $\alpha>0, \beta>0$. When $\alpha<0, \beta<0$, one can get the similar results by using the corresponding absolute values $|\alpha|$ and $|\beta|$ instead of $\alpha, \beta$.

In what follows, we present some mathematical results related to the topic of this paper. Liu and Qiao [29] obtained some interesting solutions including explicit single pseudo-peakons, two-peakon, and N-peakon solutions. Detailed dynamical interactions for two-pseudo-peakons and three-pseudo-peakons were also investigated in their paper with numerical simulations. There have been extensive studies on high order Camassa-Holm type equations in the mathematics physics fields [5,16,17,20,22,31,38-41]. For the case $\alpha=\beta=1$, on the circle, McLachlan and Zhang [31] established the local well-posedness of the solution in $H^{s}$ with $s>\frac{7}{2}$, it was shown that system $(1.1)$ with $\alpha=\beta=1$ does't admit finite time blow-up solutions. Tang and Liu [38] proved that the Cauchy problem for this equation is locally well-posed in the critical Besov space $B_{2,1}^{7 / 2}$ or $B_{p, r}^{s}\left(1 \leq p, r \leq+\infty\right.$ and $\left.s>\max \left\{3+\frac{1}{p}, \frac{7}{2}\right\}\right)$. The peakon-like solution and ill-posedness was also studied in [38]. For the case $b=2, m=u-u_{x x}+u_{x x x x}$, by using the Kato's theory, the local well-posedness [39] was studied in the Sobolev space $H^{s}$ with $s>\frac{9}{2}$. Ding [16,17] investigated the stationary solution, generality mild traveling solutions and conservative solution. Coclite, Holden and Karlsen [5] established the existence of global weak solutions. They also presented some invariant spaces under the action of the equation. In [20], the infinite propagation speed was considered for the case $m=4 u-5 u_{x x}+u_{x x x x}$. They also proved asymptotic behavior of the solution under the condition that the initial data decays exponentially and algebraically.

When $\beta=0$ (or $\alpha=0$ ), it means $m=u-\alpha^{2} u_{x x}$. The Camassa-Holm equation, the Degasperis-Procesi equation, and the Holm-Staley b-family equations are the special cases of equation (1.1) with $b=2, b=3$ and $b \in \mathbb{R}$, respectively. These equations arise at various levels 
of approximation in shallow water theory, and possess a physics background with shallow water propagation, the bi-Hamiltonian structure, Lax pair, and explicit solutions including classical soliton, cuspon, and peakon solutions.

In 1993, Camassa and Holm [3] derived an integrable shallow water equation with peaked solitons, which was called the Camassa-Holm equation. In 1999, Degasperis and Procesi [15] extended the Camassa-Holm equation to a new water wave equation (Degasperis-Procesi equation). Both Camassa-Holm equation and Degasperis-Procesi equation have attracted much attention. They are completely integrable $[11,12,14,35]$. Infinitely many conservation laws have been shown in [14,27,37]. For the Camassa-Holm equation, in [9,28], They proved the local well-posedness for the initial datum in $H^{s}$ with $s>3 / 2$. There were many works to study the blow-up phenomenon, such as [8-10,24,28,32]. McKean [32] (See also [24] for a simple proof) proved that if and only if some portion of the positive part of $y_{0}=u_{0}-u_{0 x x}$ lies to the left of some portion of its negative part, then the Camassa-Holm equation blow-up in finite time. The hierarchy properties, related finite-dimensional constrained flows, and algebro-geometric solutions of the Camassa-Holm equation were proposed in [34]. In [1], they studied the global conservative solution for the Camassa-Holm equation. Global dissipative solution have been shown in [2]. Constantin and Strauss [13] studied the orbital stability of the peakons. Himonas and his collaborators [21] obtained the persistence properties and unique continuation of solutions of the Camassa-Holm equation. In [25], the authors deduced the limit of the support of momentum density as $t$ goes to $+\infty$. In $[4,6,7,23,26,30,33,35,36,42]$, they have investigated some mathematical properties for the Degasperis-Procesi equation. For the Holm-Staley b-family equation, mathematical studies have also been presented in $[18,19,43]$.

The paper is organized as follows. In Section 2, we establish the local well-posedness and blow up scenario for the FOCH model. Conditions for global existence are found in Section 3. In Section 4, we establish the property of the infinite propagation speed for the FOCH model. In Section 5, we discuss the long time behavior for the support of momentum density of the FOCH model.

\section{LOCAL WELL-POSEDNESS AND BLOW UP SCENARIO}

Similar to the Camassa-Holm equation [9], we can establish the following local well-posedness theorem for the FOCH model (1.1).

Theorem 2.1. Let $u_{0} \in H^{s}(\mathbb{R})$ with $s>\frac{7}{2}$. Then there exist a $T>0$ depending on $\left\|u_{0}\right\|_{H^{s}}$, such that the FOCH model (1.1) has a unique solution

$$
u \in C\left([0, T) ; H^{s}(\mathbb{R})\right) \cap C^{1}\left([0, T) ; H^{s-1}(\mathbb{R})\right) .
$$

Morever, the map $u_{0} \in H^{s} \rightarrow u \in C\left([0, T) ; H^{s}(\mathbb{R})\right) \cap C^{1}\left([0, T) ; H^{s-1}(\mathbb{R})\right)$ is continuous.

The proof is similar to that of Theorem 2.1 in $[9,39]$. To make the paper concise, we would like to omit the detail proof here. The maximum value of $T$ in Theorem 2.1 is called the lifespan of the solution, in general. If $T<\infty$, that is

$$
\lim _{t \rightarrow T^{-}}\|u\|_{H^{s}}=\infty
$$

we say the solution blows up in finite time.

Before going to the blow up scenario, we have the following Lemma.

Lemma 2.2. As $m=\left(1-\alpha^{2} \partial_{x}^{2}\right)\left(1-\beta^{2} \partial_{x}^{2}\right) u$, then

$$
u=p * m, \quad p=\frac{\alpha^{2}}{\alpha^{2}-\beta^{2}} p_{1}-\frac{\beta^{2}}{\alpha^{2}-\beta^{2}} p_{2},
$$

where $p_{1}=\frac{1}{2 \alpha} e^{-\frac{|x|}{\alpha}}, p_{2}=\frac{1}{2 \beta} e^{-\frac{|x|}{\beta}}, \alpha \neq \beta, \alpha>0, \beta>0$.

Proof. Taking fourier transform to $m=\left(1-\alpha^{2} \partial_{x}^{2}\right)\left(1-\beta^{2} \partial_{x}^{2}\right) u$, we have

$$
\hat{m}=\left(1+\alpha^{2} \xi^{2}\right)\left(1+\beta^{2} \xi^{2}\right) \hat{u} .
$$

Notice that when $f(x)=e^{-a|x|}, a>0$ then

$$
\hat{f}(\xi)=\frac{2 a}{\xi^{2}+a^{2}} .
$$

It follows that

$$
\hat{u}(\xi)=\frac{1}{1+\alpha^{2} \xi^{2}} \cdot \frac{1}{1+\beta^{2} \xi^{2}} \cdot \hat{m}(\xi)=\hat{p_{1}}(\xi) \cdot \hat{p_{2}}(\xi) \cdot \hat{m}(\xi),
$$

where $p_{1}=\frac{1}{2 \alpha} e^{-\frac{|x|}{\alpha}}, p_{2}=\frac{1}{2 \beta} e^{-\frac{|x|}{\beta}}$. Then,

$$
u(x)=\mathcal{F}^{-1}\left(\hat{p_{1}}(\xi) \cdot \hat{p_{2}}(\xi) \cdot \hat{m}(\xi)\right)=p_{1} * p_{2} * m(x) .
$$


Let $p=p_{1} * p_{2}$, we have

$$
\begin{aligned}
p(x) & =\frac{1}{4 \alpha \beta} \int_{\mathbb{R}} e^{-\frac{|x-y|}{\alpha}} e^{-\frac{|y|}{\beta}} d y \\
& =\frac{1}{2\left(\alpha^{2}-\beta^{2}\right)}\left[\alpha e^{-\frac{|x|}{\alpha}}-\beta e^{-\frac{|x|}{\beta}}\right] \\
& =\frac{\alpha^{2}}{\left(\alpha^{2}-\beta^{2}\right)} p_{1}-\frac{\beta^{2}}{\left(\alpha^{2}-\beta^{2}\right)} p_{2}
\end{aligned}
$$

By Lemma 2.2, we can rewrite $u(x, t)$ as

$$
\begin{aligned}
u= & {\left[\frac{\alpha^{2}}{\left(\alpha^{2}-\beta^{2}\right)} p_{1}-\frac{\beta^{2}}{\left(\alpha^{2}-\beta^{2}\right)} p_{2}\right] * m } \\
= & \frac{\alpha}{2\left(\alpha^{2}-\beta^{2}\right)}\left[e^{-\frac{x}{\alpha}} \int_{-\infty}^{x} e^{\frac{\xi}{\alpha}} m(\xi, t) d \xi+e^{\frac{x}{\alpha}} \int_{x}^{+\infty} e^{-\frac{\xi}{\alpha}} m(\xi, t) d \xi\right] \\
& -\frac{\beta}{2\left(\alpha^{2}-\beta^{2}\right)}\left[e^{-\frac{x}{\beta}} \int_{-\infty}^{x} e^{\frac{\xi}{\beta}} m(\xi, t) d \xi+e^{\frac{x}{\beta}} \int_{x}^{+\infty} e^{-\frac{\xi}{\beta}} m(\xi, t) d \xi\right] .
\end{aligned}
$$

Differentiating $u$ with respect to $x$, we have

$$
\begin{aligned}
u_{x}= & \frac{1}{2\left(\alpha^{2}-\beta^{2}\right)}\left[-e^{-\frac{x}{\alpha}} \int_{-\infty}^{x} e^{\frac{\xi}{\alpha}} m(\xi, t) d \xi+e^{\frac{x}{\alpha}} \int_{x}^{+\infty} e^{-\frac{\xi}{\alpha}} m(\xi, t) d \xi\right] \\
& +\frac{1}{2\left(\alpha^{2}-\beta^{2}\right)}\left[e^{-\frac{x}{\beta}} \int_{-\infty}^{x} e^{\frac{\xi}{\beta}} m(\xi, t) d \xi-e^{\frac{x}{\beta}} \int_{x}^{+\infty} e^{-\frac{\xi}{\beta}} m(\xi, t) d \xi\right] .
\end{aligned}
$$

Then, we present the precise blow-up scenario.

Theorem 2.3. Assume that $u_{0} \in H^{4}(\mathbb{R})$ and let $T$ be the maximal existence time of the solution $u(x, t)$ to equation $(1.1), \alpha \neq \beta, \alpha>0, \beta>0$ with the initial data $u_{0}(x)$.

(1). If $b>\frac{1}{2}$, then the corresponding solution of the FOCH model (1.1) blows up in finite time if and only if

$$
\lim _{t \rightarrow T} \inf _{x \in \mathbb{R}}\left\{u_{x}(x, t)\right\}=-\infty
$$

(2). If $b<\frac{1}{2}$, then the corresponding solution of the FOCH model (1.1) blows up in finite time if and only if

$$
\lim _{t \rightarrow T} \sup _{x \in \mathbb{R}}\left\{u_{x}(x, t)\right\}=+\infty .
$$

Proof. By direct calculation, we have

$$
\begin{aligned}
\|m\|_{L^{2}}^{2} & =\int_{\mathbb{R}}\left[u-\left(\alpha^{2}+\beta^{2}\right) u_{x x}+\alpha^{2} \beta^{2} u_{x x x x}\right]^{2} d x \\
& =\int_{\mathbb{R}} u^{2}+\left(\alpha^{2}+\beta^{2}\right)^{2} u_{x x}^{2}-2\left(\alpha^{2}+\beta^{2}\right) u u_{x x}+\alpha^{4} \beta^{4} u_{x x x x}^{2}+2 \alpha^{2} \beta^{2} u u_{x x x x}-2\left(\alpha^{2}+\beta^{2}\right) \alpha^{2} \beta^{2} u_{x x} u_{x x x x} d x \\
& =\int_{\mathbb{R}} u^{2}+\left(\alpha^{2}+\beta^{2}\right)^{2} u_{x x}^{2}+2\left(\alpha^{2}+\beta^{2}\right) u_{x}^{2}+\alpha^{4} \beta^{4} u_{x x x x}^{2}+2 \alpha^{2} \beta^{2} u_{x x}^{2}+2\left(\alpha^{2}+\beta^{2}\right) \alpha^{2} \beta^{2} u_{x x x}^{2} d x .
\end{aligned}
$$

Hence

$$
c\|u\|_{H^{4}}^{2} \leq\|m\|_{L^{2}}^{2} \leq C\|u\|_{H^{4}}^{2},
$$

where $c$ and $C$ are positive constants depending on $\alpha$ and $\beta$. If $b>\frac{1}{2}$, direct calculation we have

If

$$
\frac{d}{d t} \int_{\mathbb{R}} m^{2} d x=(1-2 b) \int_{\mathbb{R}} u_{x} m^{2} d x \leq(1-2 b) \inf _{x \in \mathbb{R}}\left\{u_{x}(x, t)\right\} \int_{\mathbb{R}} m^{2} d x .
$$

$$
\inf _{x \in \mathbb{R}}\left\{u_{x}(x, t)\right\} \geq-M,
$$

then

$$
\frac{d}{d t} \int_{\mathbb{R}} m^{2} d x \leq-(1-2 b) M \int_{\mathbb{R}} m^{2} d x
$$


By using the Gronwall inequality,

$$
\|m\|_{L^{2}}^{2}=\int_{\mathbb{R}} m^{2} d x \leq e^{-(1-2 b) M} \int_{\mathbb{R}} m_{0}^{2} d x=e^{-(1-2 b) M}\left\|m_{0}\right\|_{L^{2}}^{2} .
$$

Therefore the $H^{4}$-norm of the solution is bounded on $[0, T)$.

On the other hand,

$$
\begin{aligned}
u & =\frac{\alpha^{2}}{\left(\alpha^{2}-\beta^{2}\right)} p_{1} * m-\frac{\beta^{2}}{\left(\alpha^{2}-\beta^{2}\right)} p_{2} * m \\
& =\frac{\alpha^{2}}{\left(\alpha^{2}-\beta^{2}\right)} \int_{\mathbb{R}} p_{1}(x-\xi) m(\xi) d \xi-\frac{\beta^{2}}{\left(\alpha^{2}-\beta^{2}\right)} \int_{\mathbb{R}} p_{2}(x-\xi) m(\xi) d \xi
\end{aligned}
$$

By the Sobolev's embedding $\left\|u_{x}\right\|_{\infty} \leq\|u\|_{H^{4}}$, it tells us if $H^{4}$-norm of the solution is bounded, then the $L^{\infty}$-norm of the first derivative is bounded.

By the same argument, we can get the similar result for $b<\frac{1}{2}$. So, we omit the details and complete the proof of Theorem 2.3 .

\section{GLOBAL EXISTENCE}

In this section, we study the global existence. Before going to our main results, we give the particle trajectory as

$$
\left\{\begin{array}{l}
q_{t}=u(q, t), \quad 0<t<T, x \in \mathbb{R}, \\
q(x, 0)=x, \quad x \in \mathbb{R},
\end{array}\right.
$$

where $T$ is the lifespan of the solution. Taking derivative (3.1) with respect to $x$, we obtain

$$
\frac{d q_{t}}{d x}=q_{t x}=u_{x}(q, t) q_{x}, \quad t \in(0, T)
$$

Therefore

$$
\left\{\begin{array}{l}
q_{x}=\exp \left\{\int_{0}^{t} u_{x}(q, s) d s\right\}, \quad 0<t<T, \quad x \in \mathbb{R}, \\
q_{x}(x, 0)=1, \quad x \in \mathbb{R},
\end{array}\right.
$$

which is always positive before the blow-up time. Therefore, the function $q(x, t)$ is an increasing diffeomorphism of the line before blow-up. In fact, direct calculation yields

$$
\frac{d}{d t}\left(m(q) q_{x}^{b}\right)=\left[m_{t}(q)+u(q, t) m_{x}(q)+b u_{x}(q, t) m(q)\right] q_{x}^{b}=0
$$

Hence, we have the following identity

$$
m(q) q_{x}^{b}=m_{0}(x), \quad 0<t<T, x \in \mathbb{R} .
$$

Theorem 3.1. Assume that $u_{0} \in H^{4}(\mathbb{R}), \alpha \neq \beta, \alpha>0, \beta>0$, if $b=\frac{1}{2}$ or $b=2$, then the corresponding solution of FOCH model (1.1) will exist globally in time.

Remark 3.1. If $\alpha=0$ or $\beta=0$, system (1.1) reduce to the well-known $b$-family equation. The global existence for $b=\frac{1}{2}$ and Theorem 3.2 can be reduce to the results for $b$-family equation [18]. The global existence for $b=2$ is the new discovery compared to the $b$-family equation.

Proof. Let

$$
E(t)=\int_{\mathbb{R}} u^{2}+\left(\alpha^{2}+\beta^{2}\right) u_{x}^{2}+\alpha^{2} \beta^{2} u_{x x}^{2} d x
$$

Differentiating $E(t)$, we have

$$
\begin{aligned}
\frac{d}{d t} E(t) & =\int_{\mathbb{R}} 2 u u_{t}+2\left(\alpha^{2}+\beta^{2}\right) u_{x} u_{x t}+2 \alpha^{2} \beta^{2} u_{x x} u_{x x t} d x \\
& =\int_{\mathbb{R}} 2 u u_{t}-2\left(\alpha^{2}+\beta^{2}\right) u u_{x x t}+2 \alpha^{2} \beta^{2} u u_{x x x x t} d x \\
& =2 \int_{\mathbb{R}} u m_{t} d x \\
& =(b-2) \int_{\mathbb{R}} u^{2} m_{x} d x
\end{aligned}
$$


It yields that $E(t)=E(0)$ when $b=2$. By the Sobolev's imbedding, we have

$$
\left\|u_{x}\right\|_{L^{\infty}} \leq\|u\|_{H^{2}}^{2} \leq C E(t)=C E(0)
$$

The global existence for $b=2$ is completed by Theorem 2.3. Applying $m$ on (1.1) and integration by parts, we obtain

$$
\begin{aligned}
\frac{d}{d t} \int_{\mathbb{R}} m^{2} d x & =-2 \int_{\mathbb{R}} b u_{x} m^{2}+m m_{x} u d x \\
& =-2 \int_{\mathbb{R}} b u_{x} m^{2}-\frac{m^{2}}{2} u_{x} d x \\
& =(1-2 b) \int_{\mathbb{R}} u_{x} m^{2} d x
\end{aligned}
$$

If $b=\frac{1}{2}$, then $\frac{d}{d t} \int_{\mathbb{R}} m^{2} d x=0$. Hence,

$$
\|u\|_{H^{4}}^{2} \leq\|m\|_{L^{2}}^{2}=\left\|m_{0}\right\|_{L^{2}}^{2} \text {. }
$$

It follows that the corresponding solution of FOCH model (1.1) exists globally when $b=\frac{1}{2}$.

Theorem 3.2. Supposing that $u_{0} \in H^{4}, \alpha \neq \beta, \alpha>0, \beta>0, m_{0}=\left(1-\alpha^{2} \partial_{x}^{2}\right)\left(1-\beta^{2} \partial_{x}^{2}\right) u_{0}$ does not change sign. Then the corresponding solution to (1.1) exists globally.

Proof. We can assume that $m_{0} \geq 0$. It is sufficient to prove $u_{x}$ is bounded for all $t$. In fact,

$$
\begin{aligned}
u_{x}= & \frac{1}{2\left(\alpha^{2}-\beta^{2}\right)}\left[e^{\frac{x}{\alpha}} \int_{x}^{+\infty} e^{-\frac{\xi}{\alpha}} m(\xi, t) d \xi-e^{-\frac{x}{\alpha}} \int_{-\infty}^{x} e^{\frac{\xi}{\alpha}} m(\xi, t) d \xi\right] \\
& +\frac{1}{2\left(\alpha^{2}-\beta^{2}\right)}\left[e^{-\frac{x}{\beta}} \int_{-\infty}^{x} e^{\frac{\xi}{\beta}} m(\xi, t) d \xi-e^{\frac{x}{\beta}} \int_{x}^{+\infty} e^{-\frac{\xi}{\beta}} m(\xi, t) d \xi\right] .
\end{aligned}
$$

If $m_{0} \geq 0, \alpha>\beta>0$, then

$$
\begin{aligned}
u_{x}= & \frac{1}{2\left(\alpha^{2}-\beta^{2}\right)}\left[e^{\frac{x}{\alpha}} \int_{x}^{+\infty} e^{-\frac{\xi}{\alpha}} m(\xi, t) d \xi-e^{-\frac{x}{\alpha}} \int_{-\infty}^{x} e^{\frac{\xi}{\alpha}} m(\xi, t) d \xi\right] \\
& +\frac{1}{2\left(\alpha^{2}-\beta^{2}\right)}\left[e^{-\frac{x}{\beta}} \int_{-\infty}^{x} e^{\frac{\xi}{\beta}} m(\xi, t) d \xi-e^{\frac{x}{\beta}} \int_{x}^{+\infty} e^{-\frac{\xi}{\beta}} m(\xi, t) d \xi\right] \\
\leq & \frac{1}{2\left(\alpha^{2}-\beta^{2}\right)}\left[e^{\frac{x}{\alpha}} \int_{x}^{\infty} e^{-\frac{\xi}{\alpha}} m(\xi, t) d \xi+e^{-\frac{x}{\beta}} \int_{-\infty}^{x} e^{\frac{\xi}{\beta}} m(\xi, t) d \xi\right] \\
\leq & \frac{1}{2\left(\alpha^{2}-\beta^{2}\right)}\left[e^{\frac{x}{\alpha}} e^{-\frac{x}{\alpha}} \int_{x}^{\infty} m(\xi, t) d \xi+e^{-\frac{x}{\beta}} e^{\frac{x}{\beta}} \int_{-\infty}^{x} m(\xi, t) d \xi\right] \\
\leq & \frac{1}{2\left(\alpha^{2}-\beta^{2}\right)}\left[\int_{\mathbb{R}} m(\xi, t) d \xi+\int_{\mathbb{R}} m(\xi, t) d \xi\right] \\
= & \frac{1}{\left(\alpha^{2}-\beta^{2}\right)} \int_{\mathbb{R}} m_{0}(\xi, t) d \xi
\end{aligned}
$$

and

$$
\begin{aligned}
u_{x}= & \frac{1}{2\left(\alpha^{2}-\beta^{2}\right)}\left[e^{\frac{x}{\alpha}} \int_{x}^{\infty} e^{-\frac{\xi}{\alpha}} m(\xi, t) d \xi-e^{-\frac{x}{\alpha}} \int_{-\infty}^{x} e^{\frac{\xi}{\alpha}} m(\xi, t) d \xi\right] \\
& +\frac{1}{2\left(\alpha^{2}-\beta^{2}\right)}\left[e^{-\frac{x}{\beta}} \int_{-\infty}^{x} e^{\frac{\xi}{\beta}} m(\xi, t) d \xi-e^{\frac{x}{\beta}} \int_{x}^{+\infty} e^{-\frac{\xi}{\beta}} m(\xi, t) d \xi\right] \\
\geq & \frac{1}{2\left(\alpha^{2}-\beta^{2}\right)}\left[-e^{-\frac{x}{\alpha}} \int_{-\infty}^{x} e^{\frac{\xi}{\alpha}} m(\xi, t) d \xi-e^{\frac{x}{\beta}} \int_{x}^{+\infty} e^{-\frac{\xi}{\beta}} m(\xi, t) d \xi\right] \\
\geq & -\frac{1}{\left(\alpha^{2}-\beta^{2}\right)} \int_{\mathbb{R}} m(\xi, t) d \xi \\
= & -\frac{1}{\left(\alpha^{2}-\beta^{2}\right)} \int_{\mathbb{R}} m_{0}(\xi, t) d \xi
\end{aligned}
$$

That is

$$
\left|u_{x}\right| \leq \frac{1}{\left(\alpha^{2}-\beta^{2}\right)} \int_{\mathbb{R}} m_{0}(\xi, t) d \xi
$$


If $m_{0} \geq 0,0<\alpha<\beta$, then

$$
\begin{aligned}
u_{x}= & \frac{1}{2\left(\alpha^{2}-\beta^{2}\right)}\left[e^{\frac{x}{\alpha}} \int_{x}^{\infty} e^{-\frac{\xi}{\alpha}} m(\xi, t) d \xi-e^{-\frac{x}{\alpha}} \int_{-\infty}^{x} e^{\frac{\xi}{\alpha}} m(\xi, t) d \xi\right] \\
& +\frac{1}{2\left(\alpha^{2}-\beta^{2}\right)}\left[e^{-\frac{x}{\beta}} \int_{-\infty}^{x} e^{\frac{\xi}{\beta}} m(\xi, t) d \xi-e^{\frac{x}{\beta}} \int_{x}^{+\infty} e^{-\frac{\xi}{\beta}} m(\xi, t) d \xi\right] \\
\leq & \frac{1}{2\left(\alpha^{2}-\beta^{2}\right)}\left[-e^{-\frac{x}{\alpha}} \int_{-\infty}^{x} e^{\frac{\xi}{\alpha}} m(\xi, t) d \xi-e^{\frac{x}{\beta}} \int_{x}^{+\infty} e^{-\frac{\xi}{\beta}} m(\xi, t) d \xi\right] \\
\leq & \frac{1}{2\left(\alpha^{2}-\beta^{2}\right)}\left[-e^{-\frac{x}{\alpha}} e^{\frac{x}{\alpha}} \int_{-\infty}^{x} m(\xi, t) d \xi-e^{\frac{x}{\beta}} e^{-\frac{x}{\beta}} \int_{x}^{\infty} m(\xi, t) d \xi\right] \\
\leq & \frac{1}{2\left(\beta^{2}-\alpha^{2}\right)}\left[\int_{\mathbb{R}} m(\xi, t) d \xi+\int_{\mathbb{R}} m(\xi, t) d \xi\right] \\
= & \frac{1}{\left(\beta^{2}-\alpha^{2}\right)} \int_{\mathbb{R}} m_{0}(\xi, t) d \xi
\end{aligned}
$$

and

$$
\begin{aligned}
u_{x}= & \frac{1}{2\left(\alpha^{2}-\beta^{2}\right)}\left[e^{\frac{x}{\alpha}} \int_{x}^{\infty} e^{-\frac{\xi}{\alpha}} m(\xi, t) d \xi-e^{-\frac{x}{\alpha}} \int_{-\infty}^{x} e^{\frac{\xi}{\alpha}} m(\xi, t) d \xi\right] \\
& +\frac{1}{2\left(\alpha^{2}-\beta^{2}\right)}\left[e^{-\frac{x}{\beta}} \int_{-\infty}^{x} e^{\frac{\xi}{\beta}} m(\xi, t) d \xi-e^{\frac{x}{\beta}} \int_{x}^{+\infty} e^{-\frac{\xi}{\beta}} m(\xi, t) d \xi\right] \\
\geq & \frac{1}{2\left(\alpha^{2}-\beta^{2}\right)}\left[e^{\frac{x}{\alpha}} \int_{x}^{\infty} e^{-\frac{\xi}{\alpha}} m(\xi, t) d \xi+e^{-\frac{x}{\beta}} \int_{-\infty}^{x} e^{\frac{\xi}{\beta}} m(\xi, t) d \xi\right] \\
\geq & -\frac{1}{\left(\beta^{2}-\alpha^{2}\right)} \int_{\mathbb{R}} m(\xi, t) d \xi \\
= & -\frac{1}{\left(\beta^{2}-\alpha^{2}\right)} \int_{\mathbb{R}} m_{0}(\xi, t) d \xi .
\end{aligned}
$$

That is

$$
\left|u_{x}\right| \leq \frac{1}{\left(\beta^{2}-\alpha^{2}\right)} \int_{\mathbb{R}} m_{0}(\xi, t) d \xi
$$

When $m_{0} \leq 0$, via the similar approach that is used above, we could also obtain the global existence result. So, we omit the details and complete the proof of Theorem 3.2.

\section{INFINITE PROPAGATION SPEED}

The main theorem reads as follows:

Theorem 4.1. Assume that the initial datum $u_{0}(x) \in H^{4}(\mathbb{R})$ is compactly supported in $[a, c]$, then for $t \in(0, T)$, the corresponding solution $u(x, t)$ to the FOCH model (1.1) $\alpha \neq \beta, \alpha>0, \beta>0$ has the following property:

$$
u(x, t)= \begin{cases}\frac{\alpha}{2\left(\alpha^{2}-\beta^{2}\right)} e^{-\frac{x}{\alpha}} E_{1}(t)-\frac{\beta}{2\left(\alpha^{2}-\beta^{2}\right)} e^{-\frac{x}{\beta}} E_{2}(t), & \text { as } x>q(c, t), \\ \frac{\alpha}{2\left(\alpha^{2}-\beta^{2}\right)} e^{\frac{x}{\alpha}} F_{1}(t)-\frac{\beta}{2\left(\alpha^{2}-\beta^{2}\right)} e^{\frac{x}{\beta}} F_{2}(t), & \text { as } x<q(a, t),\end{cases}
$$

where

$$
E_{1}(t)=\int_{\mathbb{R}} e^{\frac{x}{\alpha}} m(x, t) d x, \quad F_{1}(t)=\int_{\mathbb{R}} e^{-\frac{x}{\alpha}} m(x, t) d x
$$

and

$$
E_{2}(t)=\int_{\mathbb{R}} e^{\frac{x}{\beta}} m(x, t) d x, \quad F_{2}(t)=\int_{\mathbb{R}} e^{-\frac{x}{\beta}} m(x, t) d x,
$$

denote continuous nonvanishing functions.

Furthermore, if $\alpha>0,0<\beta \leq \sqrt{\frac{3}{2}} \alpha, 0 \leq b \leq \min \left\{3-\frac{2 \beta^{2}}{\alpha^{2}}, \frac{5}{3}\right\}, E_{1}(t)$ is strictly increasing function, while $F_{1}(t)$ is strictly decreasing function. Similarly, if $\beta>0,0<\alpha \leq \sqrt{\frac{3}{2}} \beta, 0 \leq b \leq \min \left\{3-\frac{2 \alpha^{2}}{\beta^{2}}, \frac{5}{3}\right\}, E_{2}(t)$ is strictly increasing function, while $F_{2}(t)$ is strictly decreasing function. 
Remark 4.1. Theorem 4.1 implies that the strong solution $u(x, t)$ doesn't have compact $x$-support for any $t>0$ in its lifespan, although the corresponding $u_{0}(x)$ is compactly supported.

Proof. Since $u_{0}(x)$ has a compact support in the interval $[a, c]$, so does $m_{0}(x)=\left(1-\alpha^{2} \partial_{x}^{2}\right)\left(1-\beta^{2} \partial_{x}^{2}\right) u_{0}(x)$. Equation $(3.2)$ tells us that $m(x)=\left(1-\alpha^{2} \partial_{x}^{2}\right)\left(1-\beta^{2} \partial_{x}^{2}\right) u(x)$ is compactly supported in the interval $[q(a, t), q(c, t)]$ in its lifespan. Hence the following functions are well-defined

$$
\begin{array}{lll}
E_{1}(t)=\int_{\mathbb{R}} e^{\frac{x}{\alpha}} m(x, t) d x, & F_{1}(t)=\int_{\mathbb{R}} e^{-\frac{x}{\alpha}} m(x, t) d x, \\
E_{2}(t)=\int_{\mathbb{R}} e^{\frac{x}{\beta}} m(x, t) d x, & F_{2}(t)=\int_{\mathbb{R}} e^{-\frac{x}{\beta}} m(x, t) d x .
\end{array}
$$

Using (3.2),

$$
m(q(x, t), t) \equiv 0, \quad x<a \quad \text { or } \quad x>c,
$$

we know

$$
\begin{aligned}
u(x, t) & =\left(\frac{\alpha^{2}}{\left(\alpha^{2}-\beta^{2}\right)} p_{1}-\frac{\beta^{2}}{\left(\alpha^{2}-\beta^{2}\right)} p_{2}\right) * m(x, t) \\
& =\frac{\alpha}{2\left(\alpha^{2}-\beta^{2}\right)} \int_{\mathbb{R}} e^{-\frac{|x-\xi|}{\alpha}} m(\xi) d \xi-\frac{\beta}{2\left(\alpha^{2}-\beta^{2}\right)} \int_{\mathbb{R}} e^{-\frac{|x-\xi|}{\beta}} m(\xi) d \xi \\
& =\frac{\alpha}{2\left(\alpha^{2}-\beta^{2}\right)} \int_{q(a, t)}^{q(c, t)} e^{-\frac{|x-\xi|}{\alpha}} m(\xi) d \xi-\frac{\beta}{2\left(\alpha^{2}-\beta^{2}\right)} \int_{q(a, t)}^{q(c, t)} e^{-\frac{|x-\xi|}{\beta}} m(\xi) d \xi .
\end{aligned}
$$

Then, for $x>q(c, t)$, we have

$$
\begin{aligned}
u(x, t) & =\frac{\alpha}{2\left(\alpha^{2}-\beta^{2}\right)} \int_{q(a, t)}^{q(c, t)} e^{-\frac{x-\xi}{\alpha}} m(\xi) d \xi-\frac{\beta}{2\left(\alpha^{2}-\beta^{2}\right)} \int_{q(a, t)}^{q(c, t)} e^{-\frac{x-\xi}{\beta}} m(\xi) d \xi \\
& =\frac{\alpha}{2\left(\alpha^{2}-\beta^{2}\right)} e^{-\frac{x}{\alpha}} \int_{q(a, t)}^{q(c, t)} e^{\frac{\xi}{\alpha}} m(\xi) d \xi-\frac{\beta}{2\left(\alpha^{2}-\beta^{2}\right)} e^{-\frac{x}{\beta}} \int_{q(a, t)}^{q(c, t)} e^{\frac{\xi}{\beta}} m(\xi) d \xi \\
& =\frac{\alpha}{2\left(\alpha^{2}-\beta^{2}\right)} e^{-\frac{x}{\alpha}} E_{1}(t)-\frac{\beta}{2\left(\alpha^{2}-\beta^{2}\right)} e^{-\frac{x}{\beta}} E_{2}(t) .
\end{aligned}
$$

Similarly, when $x<q(a, t)$, we have

$$
\begin{aligned}
u(x, t) & =\frac{\alpha}{2\left(\alpha^{2}-\beta^{2}\right)} \int_{q(a, t)}^{q(c, t)} e^{\frac{x-\xi}{\alpha}} m(\xi) d \xi-\frac{\beta}{2\left(\alpha^{2}-\beta^{2}\right)} \int_{q(a, t)}^{q(c, t)} e^{\frac{x-\xi}{\beta}} m(\xi) d \xi \\
& =\frac{\alpha}{2\left(\alpha^{2}-\beta^{2}\right)} e^{\frac{x}{\alpha}} \int_{q(a, t)}^{q(c, t)} e^{-\frac{\xi}{\alpha}} m(\xi) d \xi-\frac{\beta}{2\left(\alpha^{2}-\beta^{2}\right)} e^{\frac{x}{\beta}} \int_{q(a, t)}^{q(c, t)} e^{-\frac{\xi}{\beta}} m(\xi) d \xi \\
& =\frac{\alpha}{2\left(\alpha^{2}-\beta^{2}\right)} e^{\frac{x}{\alpha}} F_{1}(t)-\frac{\beta}{2\left(\alpha^{2}-\beta^{2}\right)} e^{\frac{x}{\beta}} F_{2}(t) .
\end{aligned}
$$

On the other hand,

$$
\frac{d E_{1}(t)}{d t}=\int_{\mathbb{R}} e^{\frac{\xi}{\alpha}} m_{t}(\xi, t) d \xi
$$

It is easy to get

$$
\begin{aligned}
m_{t} & =-m_{x}-b m u_{x} \\
& =\left[\left(\alpha^{2}+\beta^{2}\right) u_{x x x}-\alpha^{2} \beta^{2} u_{x x x x x}-u_{x}\right] u-b\left[u-\left(\alpha^{2}+\beta^{2}\right) u_{x x}+\alpha^{2} \beta^{2} u_{x x x x}\right] u_{x} \\
& =\left(\alpha^{2}+\beta^{2}\right) u_{x x x} u-\alpha^{2} \beta^{2} u_{x x x x x} u-(b+1) u u_{x}+b\left(\alpha^{2}+\beta^{2}\right) u_{x x} u_{x}-b \alpha^{2} \beta^{2} u_{x x x x} u_{x} .
\end{aligned}
$$


Taking (4.3) into $\frac{d E_{1}(t)}{d t}$, we obtain

$$
\begin{aligned}
\frac{d E_{1}(t)}{d t}= & \int_{\mathbb{R}} e^{\frac{x}{\alpha}} m_{t} d x \\
= & \int_{\mathbb{R}} e^{\frac{x}{\alpha}}\left[\left(\alpha^{2}+\beta^{2}\right) u_{x x x} u-\alpha^{2} \beta^{2} u_{x x x x x} u-(b+1) u u_{x}+b\left(\alpha^{2}+\beta^{2}\right) u_{x x} u_{x}-b \alpha^{2} \beta^{2} u_{x x x x} u_{x}\right] d x \\
= & \left(\alpha^{2}+\beta^{2}\right) \int_{\mathbb{R}} e^{\frac{x}{\alpha}} u_{x x x} u d x-\alpha^{2} \beta^{2} \int_{\mathbb{R}} e^{\frac{x}{\alpha}} u_{x x x x x} u d x-(b+1) \int_{\mathbb{R}} e^{\frac{x}{\alpha}} u u_{x} d x \\
& +b\left(\alpha^{2}+\beta^{2}\right) \int_{\mathbb{R}} e^{\frac{x}{\alpha}} u_{x x} u_{x} d x-b \alpha^{2} \beta^{2} \int_{\mathbb{R}} e^{\frac{x}{\alpha}} u_{x x x x} u_{x} d x \\
= & \sum_{i=1}^{5} I_{i} .
\end{aligned}
$$

$I_{1}-I_{5}$ can be estimated as follows:

$$
\begin{aligned}
I_{1} & =\left(\alpha^{2}+\beta^{2}\right) \int_{\mathbb{R}} e^{\frac{x}{\alpha}} u_{x x x} u d x \\
& =-\frac{\alpha^{2}+\beta^{2}}{2 \alpha^{3}} \int_{\mathbb{R}} e^{\frac{x}{\alpha}} u^{2} d x+\frac{3\left(\alpha^{2}+\beta^{2}\right)}{2 \alpha} \int_{\mathbb{R}} e^{\frac{x}{\alpha}} u_{x}^{2} d x \\
I_{2} & =-\alpha^{2} \beta^{2} \int_{\mathbb{R}} e^{\frac{x}{\alpha}} u_{x x x x x} u d x \\
& =\frac{\beta^{2}}{2 \alpha^{3}} \int_{\mathbb{R}} e^{\frac{x}{\alpha}} u^{2} d x-\frac{5 \beta^{2}}{2 \alpha} \int_{\mathbb{R}} e^{\frac{x}{\alpha}} u_{x}^{2} d x+\frac{5 \alpha \beta^{2}}{2} \int_{\mathbb{R}} e^{\frac{x}{\alpha}} u_{x x}^{2} d x, \\
I_{3} & =-(b+1) \int_{\mathbb{R}} e^{\frac{x}{\alpha}} u u_{x} d x \\
& =\frac{b+1}{2 \alpha} \int_{\mathbb{R}} e^{\frac{x}{\alpha}} u^{2} d x, \\
I_{4} & =b\left(\alpha^{2}+\beta^{2}\right) \int_{\mathbb{R}} e^{\frac{x}{\alpha}} u_{x x} u_{x} d x \\
& =-\frac{b\left(\alpha^{2}+\beta^{2}\right)}{2 \alpha} \int_{\mathbb{R}} e^{\frac{x}{\alpha}} u_{x}^{2} d x, \\
I_{5} & =-b \alpha^{2} \beta^{2} \int_{\mathbb{R}} e^{\frac{x}{\alpha}} u_{x x x x} u_{x} d x \\
& =\frac{b \beta^{2}}{2 \alpha} \int_{\mathbb{R}} e^{\frac{x}{\alpha}} u_{x}^{2} d x-\frac{3 b \alpha \beta^{2}}{2} \int_{\mathbb{R}} e^{\frac{x}{\alpha}} u_{x x}^{2} d x .
\end{aligned}
$$

Combining (4.5)-(4.9) to (4.4), we have

$$
\begin{aligned}
\frac{d E_{1}(t)}{d t} & =\int_{\mathbb{R}} e^{\frac{x}{\alpha}} m_{t} d x \\
& =\frac{b}{2 \alpha} \int_{\mathbb{R}} e^{\frac{x}{\alpha}} u^{2} d x-\frac{(b-3) \alpha^{2}+2 \beta^{2}}{2 \alpha} \int_{\mathbb{R}} e^{\frac{x}{\alpha}} u_{x}^{2} d x+\frac{(5-3 b) \alpha \beta^{2}}{2} \int_{\mathbb{R}} e^{\frac{x}{\alpha}} u_{x x}^{2} d x
\end{aligned}
$$

For $\alpha>0,0<\beta \leq \sqrt{\frac{3}{2}} \alpha, 0 \leq b \leq \min \left\{3-\frac{2 \beta^{2}}{\alpha^{2}}, \frac{5}{3}\right\}$, from (4.10), $E_{1}(t)$ is strictly increasing for nontrivial solution.

Similary,

$$
\begin{aligned}
\frac{d F_{1}(t)}{d t} & =\int_{\mathbb{R}} e^{-\frac{x}{\alpha}} m_{t} d x \\
& =-\frac{b}{2 \alpha} \int_{\mathbb{R}} e^{-\frac{x}{\alpha}} u^{2} d x+\frac{(b-3) \alpha^{2}+2 \beta^{2}}{2 \alpha} \int_{\mathbb{R}} e^{-\frac{x}{\alpha}} u_{x}^{2} d x-\frac{(5-3 b) \alpha \beta^{2}}{2} \int_{\mathbb{R}} e^{-\frac{x}{\alpha}} u_{x x}^{2} d x
\end{aligned}
$$

For $\alpha>0,0<\beta \leq \sqrt{\frac{3}{2}} \alpha, 0 \leq b \leq \min \left\{3-\frac{2 \beta^{2}}{\alpha^{2}}, \frac{5}{3}\right\}$, from (4.11), $F_{1}(t)$ is strictly decreasing for nontrivial solution.

$$
\begin{aligned}
\frac{d E_{2}(t)}{d t} & =\int_{\mathbb{R}} e^{\frac{x}{\beta}} m_{t} d x \\
& =\frac{b}{2 \beta} \int_{\mathbb{R}} e^{\frac{x}{\beta}} u^{2} d x-\frac{(b-3) \beta^{2}+2 \alpha^{2}}{2 \beta} \int_{\mathbb{R}} e^{\frac{x}{\beta}} u_{x}^{2} d x+\frac{(5-3 b) \beta \alpha^{2}}{2} \int_{\mathbb{R}} e^{\frac{x}{\beta}} u_{x x}^{2} d x
\end{aligned}
$$


For $\beta>0,0<\alpha \leq \sqrt{\frac{3}{2}} \beta, 0 \leq b \leq \min \left\{3-\frac{2 \alpha^{2}}{\beta^{2}}, \frac{5}{3}\right\}$, from (4.12), $E_{2}(t)$ is strictly increasing for nontrivial solution.

$$
\begin{aligned}
\frac{d F_{2}(t)}{d t} & =\int_{\mathbb{R}} e^{-\frac{x}{\beta}} m_{t} d x \\
& =-\frac{b}{2 \beta} \int_{\mathbb{R}} e^{-\frac{x}{\beta}} u^{2} d x+\frac{(b-3) \beta^{2}+2 \alpha^{2}}{2 \beta} \int_{\mathbb{R}} e^{-\frac{x}{\beta}} u_{x}^{2} d x-\frac{(5-3 b) \beta \alpha^{2}}{2} \int_{\mathbb{R}} e^{-\frac{x}{\beta}} u_{x x}^{2} d x
\end{aligned}
$$

For $\beta>0,0<\alpha \leq \sqrt{\frac{3}{2}} \beta, 0 \leq b \leq \min \left\{3-\frac{2 \alpha^{2}}{\beta^{2}}, \frac{5}{3}\right\}$, from (4.13), $F_{2}(t)$ is strictly decreasing for nontrivial solution.

This complete the proof of Theorem 4.1.

Remark 4.2. Let

$$
u^{\prime}(x, t)=\left\{\begin{array}{ll}
\frac{\alpha}{2\left(\alpha^{2}-\beta^{2}\right)} e^{-\frac{x}{\alpha}} E_{1}(t), & \text { as } x>q(c, t), \\
\frac{\alpha}{2\left(\alpha^{2}-\beta^{2}\right)} e^{\frac{x}{\alpha}} F_{1}(t), & \text { as } x<q(a, t),
\end{array} \quad u^{\prime \prime}(x, t)= \begin{cases}\frac{\beta}{2\left(\alpha^{2}-\beta^{2}\right)} e^{-\frac{x}{\beta}} E_{2}(t), & \text { as } x>q(c, t), \\
\frac{\beta}{2\left(\alpha^{2}-\beta^{2}\right)} e^{\frac{x}{\beta}} F_{2}(t), & \text { as } x<q(a, t),\end{cases}\right.
$$

We rewrite $u=u^{\prime}-u^{\prime \prime}$, as consequences of (4.1) and (4.2), we have

$$
\begin{array}{ll}
u^{\prime}(x, t)=-\alpha u_{x}^{\prime}(x, t)=\alpha^{2} u_{x x}^{\prime}(x, t)=\frac{\alpha}{2\left(\alpha^{2}-\beta^{2}\right)} e^{-\frac{x}{\alpha}} E_{1}(t), & \text { as } x>q(c, t), \\
u^{\prime}(x, t)=\alpha u_{x}^{\prime}(x, t)=\alpha^{2} u_{x x}^{\prime}(x, t)=\frac{\alpha}{2\left(\alpha^{2}-\beta^{2}\right)} e^{\frac{x}{\alpha}} F_{1}(t), \quad & \text { as } x<q(a, t) .
\end{array}
$$

and

$$
\begin{array}{ll}
u^{\prime \prime}(x, t)=-\beta u_{x}^{\prime \prime}(x, t)=\beta^{2} u_{x x}^{\prime \prime}(x, t)=\frac{\beta}{2\left(\alpha^{2}-\beta^{2}\right)} e^{-\frac{x}{\beta}} E_{2}(t), & \text { as } x>q(c, t), \\
u^{\prime \prime}(x, t)=\beta u_{x}^{\prime \prime}(x, t)=\beta^{2} u_{x x}^{\prime \prime}(x, t)=\frac{\beta}{2\left(\alpha^{2}-\beta^{2}\right)} e^{\frac{x}{\beta}} F_{2}(t), \quad & \text { as } x<q(a, t) .
\end{array}
$$

Theorem 4.2. Suppose the initial value $u_{0}(x) \in H^{4}(\mathbb{R}), m_{0}=\left(1-\alpha^{2} \partial_{x}^{2}\right)\left(1-\beta^{2} \partial_{x}^{2}\right) u_{0}, \alpha>\beta>0$, mo doesn't change sign on $\mathbb{R}$ and $u_{0}$ has compact support in the interval $[a, c]$. Then for $t \in(0, T)$, the corresponding solution $u(x, t)$ of equation (1.1) satisfies

$$
\begin{array}{cl}
\frac{1}{2(\alpha+\beta)} e^{-\frac{x}{\alpha}}\left|E_{1}(t)\right| \leq u(x, t) \leq \frac{\alpha}{2\left(\alpha^{2}-\beta^{2}\right)} e^{-\frac{x}{\alpha}}\left|E_{1}(t)\right|, & \text { as } x>q(c, t), \\
\frac{1}{2(\alpha+\beta)} e^{\frac{x}{\alpha}}\left|F_{1}(t)\right| \leq u(x, t) \leq \frac{\alpha}{2\left(\alpha^{2}-\beta^{2}\right)} e^{\frac{x}{\alpha}}\left|F_{1}(t)\right|, & \text { as } x<q(a, t) .
\end{array}
$$

where

$$
E_{1}(t)=\int_{\mathbb{R}} e^{\frac{\xi}{\alpha}} m(\xi, t) d \xi, \quad F_{1}(t)=\int_{\mathbb{R}} e^{-\frac{\xi}{\alpha}} m(\xi, t) d \xi,
$$

denote continuous nonvanishing functions.

Remark 4.3. We assume $\alpha>\beta>0$ to get the above conclusion in Theorem 4.2, because the position of $\alpha, \beta$ is symmetric, then $\beta>\alpha>0$, we have results similar to the above conclusions about $E_{2}(t)=\int_{\mathbb{R}} e^{\frac{\xi}{\beta}} m(\xi, t) d \xi, F_{2}(t)=\int_{\mathbb{R}} e^{-\frac{\xi}{\beta}} m(\xi, t) d \xi$,

$$
\begin{aligned}
& \frac{1}{2(\alpha+\beta)} e^{-\frac{x}{\beta}}\left|E_{2}(t)\right| \leq u(x, t) \leq \frac{\beta}{2\left(\beta^{2}-\alpha^{2}\right)} e^{-\frac{x}{\beta}}\left|E_{2}(t)\right|, \quad \text { as } x>q(c, t) \text {, } \\
& \frac{1}{2(\alpha+\beta)} e^{\frac{x}{\beta}}\left|F_{2}(t)\right| \leq u(x, t) \leq \frac{\beta}{2\left(\beta^{2}-\alpha^{2}\right)} e^{\frac{x}{\beta}}\left|F_{2}(t)\right|, \quad \text { as } x<q(a, t) \text {. }
\end{aligned}
$$

Theorem 4.2 can be seem as a generalization of the result in [20]. Comparing with Theorem 4.1, it show more detailed estimation by adding the additional condition on $m_{0}$.

Proof. If $u_{0}$ has a compact support set $[a, c]$, then the corresponding $m_{0}$ also has a corresponding compact support set $[a, c]$. It is known from (3.2) that $m$ has the same compact support set $[q(a, t), q(c, t)]$. We define

$$
\begin{aligned}
& u_{1}=\left(1-\frac{\beta}{\alpha}\right) \cdot \frac{\alpha}{2\left(\alpha^{2}-\beta^{2}\right)} \int_{\mathbb{R}} e^{-\frac{|x-\xi|}{\alpha}} m d \xi=\frac{1}{2(\alpha+\beta)} \int_{\mathbb{R}} e^{-\frac{|x-\xi|}{\alpha}} m d \xi, \\
& u_{2}=\frac{\alpha}{2\left(\alpha^{2}-\beta^{2}\right)} \int_{\mathbb{R}} e^{-\frac{|x-\xi|}{\alpha}} m d \xi .
\end{aligned}
$$


According to $E_{1}(t)=\int_{\mathbb{R}} e^{\frac{\xi}{\alpha}} m(\xi, t) d \xi, F_{1}(t)=\int_{\mathbb{R}} e^{-\frac{\xi}{\alpha}} m(\xi, t) d \xi$, then

$$
\begin{aligned}
& u_{1}(x, t)=\frac{1}{2(\alpha+\beta)} e^{-\frac{x}{\alpha}} E_{1}(t), \quad u_{2}(x, t)=\frac{\alpha}{2\left(\alpha^{2}-\beta^{2}\right)} e^{-\frac{x}{\alpha}} E_{1}(t), \quad \text { as } x>q(c, t), \\
& u_{1}(x, t)=\frac{1}{2(\alpha+\beta)} e^{\frac{x}{\alpha}} F_{1}(t), \quad u_{2}(x, t)=\frac{\alpha}{2\left(\alpha^{2}-\beta^{2}\right)} e^{\frac{x}{\alpha}} F_{1}(t), \quad \text { as } x<q(a, t) .
\end{aligned}
$$

According to (4.1) and (4.14), we obtain

$$
\begin{aligned}
& u_{2}(x, t)-u(x, t)=\frac{\beta}{2\left(\alpha^{2}-\beta^{2}\right)} \int_{\mathbb{R}} e^{-\frac{|x-\xi|}{\beta}} m d \xi \\
& u(x, t)-u_{1}(x, t)=\frac{\beta}{2\left(\alpha^{2}-\beta^{2}\right)} \int_{\mathbb{R}}\left(e^{-\frac{|x-\xi|}{\alpha}}-e^{-\frac{|x-\xi|}{\beta}}\right) m d \xi .
\end{aligned}
$$

Then, we obtain

$$
\begin{cases}u_{1}(x, t) \leq u(x, t) \leq u_{2}(x, t), & m_{0} \geq 0 \\ u_{2}(x, t) \leq u(x, t) \leq u_{1}(x, t), & m_{0} \leq 0\end{cases}
$$

If $m_{0} \geq 0$,

$$
\begin{cases}\frac{1}{2(\alpha+\beta)} e^{-\frac{x}{\alpha}} E_{1}(t) \leq u(x, t) \leq \frac{\alpha}{2\left(\alpha^{2}-\beta^{2}\right)} e^{-\frac{x}{\alpha}} E_{1}(t), & \text { as } x>q(c, t), \\ \frac{1}{2(\alpha+\beta)} e^{\frac{x}{\alpha}} F_{1}(t) \leq u(x, t) \leq \frac{\alpha}{2\left(\alpha^{2}-\beta^{2}\right)} e^{\frac{x}{\alpha}} F_{1}(t), & \text { as } x<q(a, t) .\end{cases}
$$

If $m_{0} \leq 0$,

$$
\begin{cases}\frac{\alpha}{2\left(\alpha^{2}-\beta^{2}\right)} e^{-\frac{x}{\alpha}} E_{1}(t) \leq u(x, t) \leq \frac{1}{2(\alpha+\beta)} e^{-\frac{x}{\alpha}} E_{1}(t), & \text { as } x>q(c, t), \\ \frac{\alpha}{2\left(\alpha^{2}-\beta^{2}\right)} e^{\frac{x}{\alpha}} F_{1}(t) \leq u(x, t) \leq \frac{1}{2(\alpha+\beta)} e^{\frac{x}{\alpha}} F_{1}(t), & \text { as } x<q(a, t) .\end{cases}
$$

The proof of Theorem 4.2 is finished.

\section{LONG TIME BEHAVIOR FOR THE SUPPORT OF MOMENTUM DENSITY}

After the global existence of solution is established, we will discuss the long time behavior for the support of momentum density of the FOCH model. Now, we give the lemma and main theorem as follows:

Lemma 5.1. Let $\alpha>\beta>0$, Assume the initial value $u_{0} \not \equiv 0$ has a compact supported set $[a, c]$.

(1). If $m_{0}(x) \geq 0(\not \equiv 0), x \in[a, c]$, then we have

$$
\lim _{t \rightarrow+\infty} F_{1}(t)=0
$$

(2). If $m_{0}(x) \leq 0(\not \equiv 0), x \in[a, c]$, then we have

$$
\lim _{t \rightarrow+\infty} E_{1}(t)=0
$$

Remark 5.1. By the same argument, we can get a similar conclusion for $\beta>\alpha>0$. If $m_{0}(x) \geq 0(\not \equiv 0), x \in[a, c]$, then we have

$$
\lim _{t \rightarrow+\infty} F_{2}(t)=0 \text {. }
$$

If $m_{0}(x) \leq 0(\not \equiv 0), x \in[a, c]$, then we have

$$
\lim _{t \rightarrow+\infty} E_{2}(t)=0
$$

Proof. (1) For $m_{0}(x)>0$, from (3.2), we have $E_{1}(t)>0, F_{1}(t)>0, E_{2}(t)>0, F_{2}(t)>0$, for all $t \geq 0$. As $F_{1}(t)>0$, we claim that

$$
\lim _{t \rightarrow+\infty} F_{1}(t)=0 \text {. }
$$

Otherwise, there is a constant $\epsilon_{0}>0$, for any $T>0$, there will exist a $t>T$, such that $F_{1}(t) \geq \epsilon_{0}$.

For any $d<a$, from (4.15) we have

$$
\begin{aligned}
\frac{d}{d t} q(d, t)=u(q(d, t), t) & \geq \frac{1}{2(\alpha+\beta)} e^{\frac{q(d, t)}{\alpha}} F_{1}(t) \\
& \geq \frac{1}{2(\alpha+\beta)} e^{\frac{q(d, t)}{\alpha}} \epsilon_{0} .
\end{aligned}
$$


It follows that

$$
e^{-\frac{q(d, t)}{\alpha}} \leq-\frac{\epsilon_{0}}{2 \alpha(\alpha+\beta)} t+\frac{e^{-\frac{d}{\alpha}}}{\alpha}
$$

Taking $T=\frac{2(\alpha+\beta)}{\epsilon_{0}} e^{-\frac{d}{\alpha}}$, however, when $t=T+1$,

$$
-\frac{\epsilon_{0}}{2 \alpha(\alpha+\beta)} t+\frac{e^{-\frac{d}{\alpha}}}{\alpha}<0,
$$

This is the contradiction. So our claim is right.

(2). For $m_{0}(x)<0$, from (3.2), we have $E_{1}(t)<0, F_{1}(t)<0, E_{2}(t)<0, F_{2}(t)<0$, for all $t \geq 0$. As $F_{1}(t)>0$, we claim that

$$
\lim _{t \rightarrow+\infty} E_{1}(t)=0 \text {. }
$$

Otherwise, there is a constant $\epsilon_{0}>0$, for any $T>0$, for any $T>0$, there will exist a $t>T$, such that $E_{1}(t) \leq-\epsilon_{0}$.

For any $h>c$, from $(4.16)$ we have

$$
\begin{aligned}
\frac{d}{d t} q(h, t)=u(q(h, t), t) & \leq \frac{1}{2(\alpha+\beta)} e^{-\frac{q(h, t)}{\alpha}} E_{1}(t) \\
& \leq-\frac{\epsilon_{0}}{2(\alpha+\beta)} e^{-\frac{q(h, t)}{\alpha}} .
\end{aligned}
$$

It follows that

$$
e^{\frac{q(h, t)}{\alpha}} \leq-\frac{\epsilon_{0}}{2 \alpha(\alpha+\beta)} t+\frac{e^{-\frac{d}{\alpha}}}{\alpha}
$$

Taking $T=\frac{2(\alpha+\beta)}{\epsilon_{0}} e^{-\frac{d}{\alpha}}$, however, when $t=T+1$,

$$
-\frac{\epsilon_{0}}{2 \alpha(\alpha+\beta)} t+\frac{e^{-\frac{d}{\alpha}}}{\alpha}<0
$$

This is the contradiction. So our claim is right.

Theorem 5.2. If $b>1, \alpha>\beta>0$, and suppose that $m_{0}(x) \in L_{\frac{1}{b}}$ and $u_{0}(x)$ has a compact supported set $[a, c]$.

(1). If $m_{0}(x) \geq 0(\not \equiv 0), x \in[a, c]$, then we have

$$
e^{\frac{q(c, t)}{\alpha(b-1)}}-e^{\frac{q(a, t)}{\alpha(b-1)}} \longrightarrow+\infty, \quad \text { as } t \longrightarrow+\infty .
$$

(2). If $m_{0}(x) \leq 0(\not \equiv 0), x \in[a, c]$, then we have

$$
e^{-\frac{q(a, t)}{\alpha(b-1)}}-e^{-\frac{q(c, t)}{\alpha(b-1)}} \longrightarrow+\infty, \quad \text { as } t \longrightarrow+\infty .
$$

Remark 5.2. For the case $\beta>\alpha>0$, by using the properties of $E_{2}$ and $F_{2}$ in Remark 5.1, one can replace $\alpha$ with $\beta$ in (5.1) and (5.2).

Proof. (1) By (3.2) and direct calculation, we have

$$
\begin{aligned}
\left(\int_{a}^{c}\left(m_{0}\right)^{\frac{1}{b}} d x\right)^{b} & =\left(\int_{a}^{c}\left(m(q, t) q_{x}^{b}\right)^{\frac{1}{b}} d x\right)^{b}=\left(\int_{a}^{c}(m(q, t))^{\frac{1}{b}} q_{x} d x\right)^{b} \\
& =\left(\int_{q(a, t)}^{q(c, t)}(m(\xi, t))^{\frac{1}{b}} d \xi\right)^{b} \\
& \leq\left(\int_{q(a, t)}^{q(c, t)} m(\xi, t) e^{-\frac{\xi}{\alpha}} d \xi\right)\left[\int_{q(a, t)}^{q(c, t)} e^{\frac{\xi}{\alpha(b-1)}} d \xi\right]^{b-1} \\
& =F_{1}(t)\left[\alpha(b-1)\left(e^{\frac{q(c, t)}{\alpha(b-1)}}-e^{\frac{q(a, t)}{\alpha(b-1)}}\right)\right]^{(b-1)} .
\end{aligned}
$$


It follows

Using the limit

$$
\left[\alpha(b-1)\left(e^{\frac{q(c, t)}{\alpha(b-1)}}-e^{\frac{q(a, t)(b-1)}{\alpha}}\right)\right]^{(b-1)} \geq \frac{\left(\int_{a}^{c}\left(m_{0}\right)^{\frac{1}{b}} d x\right)^{b}}{F_{1}(t)} .
$$

$$
\lim _{t \rightarrow+\infty} F_{1}=0
$$

we can get

$$
e^{\frac{q(c, t)}{\alpha(b-1)}}-e^{\frac{q(a, t)}{\alpha(b-1)}} \longrightarrow+\infty, \text { as } t \longrightarrow+\infty .
$$

(2). Direct calculation, we have

$$
\begin{aligned}
\left(\int_{a}^{c}\left(-m_{0}\right)^{\frac{1}{b}} d x\right)^{b} & =\left(\int_{a}^{c}\left(-m_{0}\right)^{\frac{1}{b}} d x\right)^{b}=\left(\int_{a}^{c}\left(-m(q, t) q_{x}^{b}\right)^{\frac{1}{b}} d x\right)^{b} \\
& =\left(\int_{a}^{c}(-m(q, t))^{\frac{1}{b}} q_{x} d x\right)^{b}=\left(\int_{q(a, t)}^{q(c, t)}(-m(\xi, t))^{\frac{1}{b}} d \xi\right)^{b} \\
& \leq\left(\int_{q(a, t)}^{q(c, t)}\left(-m(\xi, t) e^{\frac{\xi}{\alpha}}\right) d \xi\right)\left[\int_{q(a, t)}^{q(c, t)} e^{-\frac{\xi}{\alpha(b-1)}} d \xi\right]^{b-1} \\
& =-E_{1}(t)\left[\alpha(b-1)\left(e^{-\frac{q(a, t)}{\alpha(b-1)}}-e^{\left.-\frac{q(c, t)}{\alpha(b-1)}\right)}\right]^{b-1} .\right.
\end{aligned}
$$

It follows

$$
\left[\alpha(b-1)\left(e^{-\frac{q(a, t)}{\alpha(b-1)}}-e^{-\frac{q(c, t)}{\alpha(b-1)}}\right)\right]^{b-1} \geq \frac{\left(\int_{a}^{c}\left(-m_{0}\right)^{\frac{1}{b}} d x\right)^{b}}{-E_{1}(t)} .
$$

Using the limit

$$
\lim _{t \rightarrow+\infty} E_{1}=0
$$

we can obtain

$$
e^{-\frac{q(a, t)}{\alpha(b-1)}}-e^{-\frac{q(c, t)}{\alpha(b-1)}} \longrightarrow+\infty, \text { as } t \longrightarrow+\infty .
$$

Theorem 5.3. If $b=1$, suppose that $m_{0}(x) \in L_{1}$ and $u_{0}(x)$ has a compact supported set $[a, c]$.

(1). If $m_{0}(x) \geq 0(\not \equiv 0), x \in[a, c]$, then we have

$$
q(c, t) \longrightarrow+\infty, \quad \text { as } t \longrightarrow+\infty \text {. }
$$

(2). If $m_{0}(x) \leq 0(\not \equiv 0), x \in[a, c]$, then we have

$$
q(a, t) \longrightarrow-\infty, \quad \text { as } t \longrightarrow+\infty .
$$

Proof. We only present the proof for $\alpha>\beta>0$. The case $\beta>\alpha>0$ can be proved by the same argument. (1) As $m_{0}(x) \geq 0$, for any $t \geq 0$, we have $F_{1}(t)>0$. According to Lemma 5.1, we know

$$
\lim _{t \rightarrow+\infty} F_{1}(t)=0
$$

Direct calculation, we have

$$
\int_{a}^{c} m_{0} d x=\int_{a}^{c} m(q, t) q_{x} d x \leq e^{\frac{q(c, t)}{\alpha}} \int_{q(a, t)}^{q(c, t)} m(\xi, t) e^{-\frac{\xi}{\alpha}} d \xi=e^{\frac{q(c, t)}{\alpha}} F_{1}(t) .
$$

It follows

$$
e^{\frac{q(c, t)}{\alpha}} \geq \frac{\int_{a}^{c} m_{0} d x}{F_{1}(t)} \longrightarrow+\infty, \quad \text { as } t \longrightarrow+\infty,
$$


then we can get

$$
q(c, t) \longrightarrow+\infty, \quad \text { as } t \longrightarrow+\infty \text {. }
$$

(2). As $m_{0}(x) \leq 0$, for any $t \geq 0$, we have $E_{1}(t)<0$. According to Lemma 5.1, we know

$$
\lim _{t \rightarrow+\infty} E_{1}(t)=0 \text {. }
$$

Direct calculation, we have

$$
\begin{aligned}
\int_{a}^{c}\left(-m_{0}\right) d x & =\int_{a}^{c}\left(-m(q, t) q_{x}\right) d x \\
& \leq e^{-\frac{q(a, t)}{\alpha}} \int_{q(a, t)}^{q(c, t)}(-m(\xi, t)) e^{\frac{\xi}{\alpha}} d \xi=-e^{-\frac{q(a, t)}{\alpha}} E_{1}(t) .
\end{aligned}
$$

It follows

$$
e^{-\frac{q(a, t)}{\alpha}} \geq \frac{\int_{a}^{c}\left(-m_{0}\right) d x}{-E_{1}(t)} \longrightarrow+\infty, \quad \text { as } t \longrightarrow+\infty,
$$

then we can get

$$
q(a, t) \longrightarrow-\infty, \quad \text { as } t \longrightarrow+\infty .
$$

Theorem 5.4. If $0<b<1, \alpha>\beta>0, m_{0}(x) \in L_{\frac{1}{b}}$ or $b=0, m_{0} \in L_{\infty}$. Suppose that $u_{0}(x)$ has a compact supported set [a,c].

(1). If $m_{0}(x) \geq 0(\not \equiv 0)$ for $x \in[a, c]$, then we have

$$
e^{\frac{q(c, t)}{\alpha}}-e^{\frac{q(a, t)}{\alpha}} \longrightarrow+\infty, \quad \text { as } t \longrightarrow+\infty .
$$

(2). If $m_{0}(x) \leq 0(\not \equiv 0)$ for $x \in[a, c]$, then we have

$$
e^{-\frac{q(a, t)}{\alpha}}-e^{-\frac{q(c, t)}{\alpha}} \longrightarrow+\infty, \text { as } t \longrightarrow+\infty .
$$

Remark 5.3. For the case $\beta>\alpha>0$, by using the properties of $E_{2}$ and $F_{2}$ in Remark 5.1, one can replace $\alpha$ with $\beta$ in (5.3) and (5.4).

Proof. (1). For $m_{0}(x) \geq 0$, we have $F_{1}(t)>0$ for all $t \geq 0$. From Lemma 5.1, we know

$$
\lim _{t \rightarrow+\infty} F_{1}(t)=0
$$

According to the conservation law

$$
\begin{gathered}
\int_{\mathbb{R}} m d x=\int_{\mathbb{R}} m_{0} d x, \quad \int_{\mathbb{R}} m^{\frac{1}{b}} d x=\int_{\mathbb{R}} m_{0}^{\frac{1}{b}} d x . \\
\text { If } 0<b<1 \text { and }\left\{\begin{array} { l } 
{ \gamma + \frac { \eta } { b } = 1 , } \\
{ 2 \gamma + \eta = 1 , } \\
{ 0 < \gamma , \eta < 1 . }
\end{array} \Longrightarrow \left\{\begin{array}{l}
0<\eta=\frac{2}{2-b}-1<1, \\
0<\gamma=1+\frac{1}{b-2}<1 .
\end{array}\right.\right.
\end{gathered}
$$

By direct calculation, we obtain

$$
\begin{aligned}
\int_{\mathbb{R}} m_{0} d x & =\int_{\mathbb{R}} m d x=\int_{\mathbb{R}} m(q, t) q_{x} d x \\
& =\left[\int_{a}^{c}\left(m e^{-\frac{q}{\alpha}} q_{x}\right)^{\gamma}\left(m^{\frac{1}{b}} q_{x}\right)^{\eta}\left(e^{\frac{q}{\alpha}} q_{x}\right)^{\gamma} d x\right] \\
& \leq\left(\int_{a}^{c} m e^{-\frac{q}{\alpha}} q_{x} d x\right)^{\gamma}\left(\int_{a}^{c} m^{\frac{1}{b}} q_{x} d x\right)^{\eta}\left(\int_{a}^{c} e^{\frac{q}{\alpha}} q_{x} d x\right)^{\gamma} \\
& =\left(\int_{q(a, t)}^{q(c, t)} m e^{-\frac{\xi}{\alpha}} d \xi\right)^{\gamma}\left(\int_{\mathbb{R}} m^{\frac{1}{b}} d \xi\right)^{\eta}\left(\int_{q(a, t)}^{q(c, t)} e^{\frac{\xi}{\alpha}} d \xi\right)^{\gamma} \\
& =\left(F_{1}(t)\right)^{\gamma}\left(\int_{\mathbb{R}} m^{\frac{1}{b}} d \xi\right)^{\eta}\left(\int_{q(a, t)}^{q(c, t)} e^{\frac{\xi}{\alpha}} d \xi\right)^{\gamma} \\
& =\left(F_{1}(t)\right)^{\gamma}\left(\int_{\mathbb{R}} m_{0}^{\frac{1}{b}} d \xi\right)^{\eta}\left(\alpha e^{\frac{q(c, t)}{\alpha}}-\alpha e^{\frac{q(a, t)}{\alpha}}\right)^{\gamma} .
\end{aligned}
$$


334

M. Thu et al. / Journal of Nonlinear Mathematical Physics 28(3) 321-336

It follows

$$
\left(\alpha e^{\frac{q(c, t)}{\alpha}}-\alpha e^{\frac{q(a, t)}{\alpha}}\right)^{\gamma} \geq \frac{\int_{\mathbb{R}} m_{0} d x}{\left(F_{1}(t)\right)^{\gamma}\left(\int_{\mathbb{R}} m_{0}^{\frac{1}{b}} d \xi\right)^{\eta}} \longrightarrow+\infty
$$

then we can obtain

$$
e^{\frac{q(c, t)}{\alpha}}-e^{\frac{q(a, t)}{\alpha}} \longrightarrow+\infty, \quad \text { as } t \longrightarrow+\infty
$$

If $b=0$, we can obtain

$$
\begin{aligned}
\int_{\mathbb{R}} m_{0} d x & =\lim _{b \rightarrow 0} \int_{\mathbb{R}} m d x=\int_{\mathbb{R}} m(q, t) q_{x} d x \\
& =\lim _{b \rightarrow 0}\left[\int_{a}^{c}\left(m e^{-\frac{q}{\alpha}} q_{x}\right)^{\gamma}\left(m^{\frac{1}{b}} q_{x}\right)^{\eta}\left(e^{\frac{q}{\alpha}} q_{x}\right)^{\gamma} d x\right] \\
& \leq \lim _{b \rightarrow 0}\left(\int_{a}^{c} m e^{-\frac{q}{\alpha}} q_{x} d x\right)^{\gamma}\left(\int_{a}^{c} m^{\frac{1}{b}} q_{x} d x\right)^{\eta}\left(\int_{a}^{c} e^{\frac{q}{\alpha}} q_{x} d x\right)^{\gamma} \\
& =\lim _{b \rightarrow 0}\left(\int_{q(a, t)}^{q(c, t)} m e^{-\frac{\xi}{\alpha}} d \xi\right)^{\gamma}\left(\int_{\mathbb{R}} m^{\frac{1}{b}} d \xi\right)^{\eta}\left(\int_{q(a, t)}^{q(c, t)} e^{\frac{\xi}{\alpha}} d \xi\right)^{\gamma} \\
& =\lim _{b \rightarrow 0}\left(F_{1}(t)\right)^{\gamma}\left(\int_{\mathbb{R}} m^{\frac{1}{b}} d \xi\right)^{\eta}\left(\int_{q(a, t)}^{q(c, t)} e^{\frac{\xi}{\alpha}} d \xi\right)^{\gamma} \\
& =\lim _{b \rightarrow 0}\left(F_{1}(t)\right)^{\gamma}\left(\int_{\mathbb{R}} m_{0}^{\frac{1}{b}} d \xi\right)^{\eta}\left(\alpha e^{\frac{q(c, t)}{\alpha}}-\alpha e^{\frac{q(a, t)}{\alpha}}\right)^{\gamma} .
\end{aligned}
$$

It follows

$$
\left(\alpha e^{\frac{q(c, t)}{\alpha}}-\alpha e^{\frac{q(a, t)}{\alpha}}\right)^{\gamma} \geq \frac{\int_{\mathbb{R}} m_{0} d x}{\left(F_{1}(t)\right)^{\gamma}\left(\lim _{b \rightarrow 0} \int_{\mathbb{R}} m_{0}^{\frac{1}{b}} d \xi\right)^{\eta}} \longrightarrow+\infty
$$

then we can obtain

$$
e^{\frac{q(c, t)}{\alpha}}-e^{\frac{q(a, t)}{\alpha}} \longrightarrow+\infty, \quad \text { as } t \longrightarrow+\infty
$$

(2). For $m_{0}(x) \leq 0$, we have $E_{1}(t)<0$ for all $t \geq 0$. From Lemma 5.1, we know

$$
\lim _{t \rightarrow+\infty} E_{1}(t)=0
$$

Similarly, according to the conservation law

$$
\begin{gathered}
\int_{\mathbb{R}} m d x=\int_{\mathbb{R}} m_{0} d x, \quad \int_{\mathbb{R}} m^{\frac{1}{b}} d x=\int_{\mathbb{R}} m_{0}^{\frac{1}{b}} d x . \\
\text { If } 0<b<1 \text { and }\left\{\begin{array}{l}
\gamma+\frac{\eta}{b}=1, \\
2 \gamma+\eta=1, \\
0<\gamma, \eta<1 .
\end{array}\right.
\end{gathered}
$$

By direct calculation, we obtain

$$
\begin{aligned}
-\int_{\mathbb{R}} m_{0} d x & =-\int_{\mathbb{R}} m d x=-\int_{\mathbb{R}} m(q, t) q_{x} d x \\
& =\left[\int_{a}^{c}\left(-m e^{\frac{q}{\alpha}} q_{x}\right)^{\gamma}\left((-m)^{\frac{1}{b}} q_{x}\right)^{\eta}\left(e^{-\frac{q}{\alpha}} q_{x}\right)^{\gamma} d x\right] \\
& \leq\left(\int_{a}^{c}-m e^{\frac{q}{\alpha}} q_{x} d x\right)^{\gamma}\left(\int_{a}^{c}(-m)^{\frac{1}{b}} q_{x} d x\right)^{\eta}\left(\int_{a}^{c} e^{-\frac{q}{\alpha}} q_{x} d x\right)^{\gamma} \\
& =\left(\int_{q(a, t)}^{q(c, t)}-m e^{\frac{\xi}{\alpha}} d \xi\right)^{\gamma}\left(\int_{\mathbb{R}}(-m)^{\frac{1}{b}} d \xi\right)^{\eta}\left(\int_{q(a, t)}^{q(c, t)} e^{-\frac{\xi}{\alpha}} d \xi\right)^{\gamma}
\end{aligned}
$$




$$
\begin{aligned}
& =\left(-E_{1}(t)\right)^{\gamma}\left(\int_{\mathbb{R}} m^{\frac{1}{b}} d \xi\right)^{\eta}\left(\int_{q(a, t)}^{q(c, t)} e^{-\frac{\xi}{\alpha}} d \xi\right)^{\gamma} \\
& =\left(-E_{1}(t)\right)^{\gamma}\left(\int_{\mathbb{R}} m_{0}^{\frac{1}{b}} d \xi\right)^{\eta}\left(\alpha e^{-\frac{q(a, t)}{\alpha}}-\alpha e^{-\frac{q(c, t)}{\alpha}}\right)^{\gamma} .
\end{aligned}
$$

It follows

$$
\left(\alpha e^{-\frac{q(a, t)}{\alpha}}-\alpha e^{-\frac{q(c, t)}{\alpha}}\right)^{\gamma} \geq \frac{-\int_{\mathbb{R}} m_{0} d x}{\left(-E_{1}(t)\right)^{\gamma}\left(\int_{\mathbb{R}}\left(-m_{0}\right)^{\frac{1}{b}} d \xi\right)^{\eta}} \longrightarrow+\infty,
$$

then we can obtain

$$
e^{-\frac{q(a, t)}{\alpha}}-e^{-\frac{q((c, t)}{\alpha}} \longrightarrow+\infty, \quad \text { as } t \longrightarrow+\infty
$$

If $b=0$, we can obtain

$$
\begin{aligned}
-\int_{\mathbb{R}} m_{0} d x & =-\lim _{b \rightarrow 0} \int_{\mathbb{R}} m d x=-\int_{\mathbb{R}} m(q, t) q_{x} d x \\
& =\lim _{b \rightarrow 0}\left[\int_{a}^{c}\left(-m e^{\frac{q}{\alpha}} q_{x}\right)^{\gamma}\left((-m)^{\frac{1}{b}} q_{x}\right)^{\eta}\left(e^{-\frac{q}{\alpha}} q_{x}\right)^{\gamma} d x\right] \\
& \leq \lim _{b \rightarrow 0}\left(\int_{a}^{c}-m e^{\frac{q}{\alpha}} q_{x} d x\right)^{\gamma}\left(\int_{a}^{c}(-m)^{\frac{1}{b}} q_{x} d x\right)^{\eta}\left(\int_{a}^{c} e^{-\frac{q}{\alpha}} q_{x} d x\right)^{\gamma} \\
& =\lim _{b \rightarrow 0}\left(\int_{q(a, t)}^{q(c, t)}-m e^{\frac{\xi}{\alpha}} d \xi\right)^{\gamma}\left(\int_{\mathbb{R}}(-m)^{\frac{1}{b}} d \xi\right)^{\eta}\left(\int_{q(a, t)}^{q(c, t)} e^{-\frac{\xi}{\alpha}} d \xi\right)^{\gamma} \\
& =\lim _{b \rightarrow 0}\left(-E_{1}(t)\right)^{\gamma}\left(\int_{\mathbb{R}} m^{\frac{1}{b}} d \xi\right)^{\eta}\left(\int_{q(a, t)}^{q(c, t)} e^{-\frac{\xi}{\alpha}} d \xi\right)^{\gamma} \\
& =\lim _{b \rightarrow 0}\left(-E_{1}(t)\right)^{\gamma}\left(\int_{\mathbb{R}} m_{0}^{\frac{1}{b}} d \xi\right)^{\eta}\left(\alpha e^{-\frac{q(a, t)}{\alpha}}-\alpha e^{-\frac{q(c, t)}{\alpha}}\right)^{\gamma} .
\end{aligned}
$$

It follows

$$
\left(\alpha e^{-\frac{q(a, t)}{\alpha}}-\alpha e^{-\frac{q(c, t)}{\alpha}}\right)^{\gamma} \geq \frac{-\int_{\mathbb{R}} m_{0} d x}{\left(-E_{1}(t)\right)^{\gamma}\left(\lim _{b \rightarrow 0} \int_{\mathbb{R}}\left(-m_{0}\right)^{\frac{1}{b}} d \xi\right)^{\eta}} \longrightarrow+\infty,
$$

then we can obtain

$$
e^{-\frac{q(a, t)}{\alpha}}-e^{-\frac{q((, t)}{\alpha}} \longrightarrow+\infty, \quad \text { as } t \longrightarrow+\infty
$$

\section{CONCLUSION}

We have considered the FOCH model $\alpha \neq \beta, \alpha>0, \beta>0$. When $\alpha, \beta$ is negative, one can get the same results by taking absolute value $|\alpha|$ and $|\beta|$. This model is highly related to the classical Camassa-Holm equation, the Degasperis-Procesi equation and the Holm-Staley b-family equation. We have studied some mathematical property, such as global existence, infinite propagation speed and long time behavior of the support of momentum density. Another highly related equation is (1.1) with $\alpha=\beta$. Due to (1.1) with $\alpha=\beta$ doesn't have the structure in Lemma 2.2 and (2.1), some results in this manuscript may can't been realized for $\alpha=\beta$.

\section{CONFLICTS OF INTEREST}

The authors declare they have no conflicts of interest.

\section{ACKNOWLEDGEMENTS}

This work has partially been supported by the National Natural Science Foundation of China (No. 12071439 and No. 11971475) and the 2019 - 2020 Hunan overseas distinguished professorship project (No. 2019014). The third author Jiang was partially supported by NSFC (grant No. 11101376, 11671309, 11671364) and ZJNSF (grant No. LY19A010016). The fourth author Qiao thanks the UT President's Endowed Professorship (Project \# 450000123) for its partial support. 


\section{REFERENCES}

[1] A. Bressan, A. Constantin, Global conservative solutions of the Camassa-Holm equation, Arch. Ration. Mech. Anal. 183 (2007), 215-239.

[2] A. Bressan, A. Constantin, Global dissipative solutions of the Camassa-Holm equation, Anal. Appl. 5 (2007), 1-27.

[3] R. Camassa, D.D. Holm, An integrable shallow water equation with peaked solitons, Phys. Rev. Lett. 71 (1993), $1661-1664$.

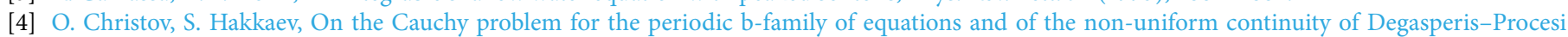
equation, J. Math. Anal. Appl. 360 (2009), 47-56.

[5] G.M. Coclite, H. Holden, K.H. Karlsen, Well-posedness of higher-order Camassa-Holm equations, J. Diff. Eqs. 246 (2009), $929-963$.

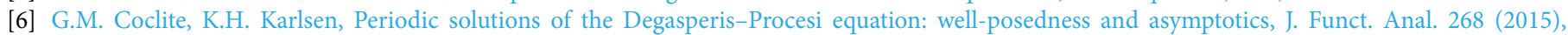
$1053-1077$.

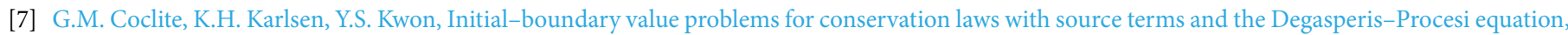
J. Funct. Anal. 257 (2009), 3823-3857.

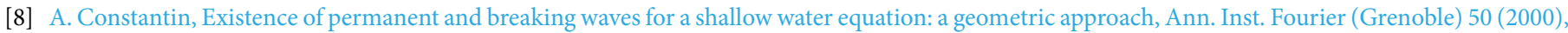
$321-362$.

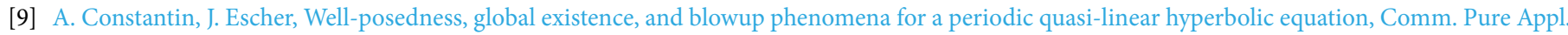
Math. 51 (1998), 475-504.

[10] A. Constantin, J. Escher, Wave breaking for nonlinear nonlocal shallow water equations, Acta Math. 181 (1998), $229-243$.

[11] A. Constantin, V.S. Gerdjikov, R.I. Ivanov, Inverse scattering transform for the Camassa-Holm equation, Inver. Prob. 22 (2006), $2197-2207$.

[12] A. Constantin, H.P. McKean, A shallow water equation on the circle, Comm. Pure Appl. Math. 52 (1999), 949-982.

[13] A. Constantin, W.A. Strauss, Stability of peakons, Comm. Pure Appl. Math. 53 (2000), 603-610.

[14] A. Degasperis, D.D. Holm, A.N.I. Khon, A new integrable equation with peakon solutions, Teoret. Mat. Fiz. 133 (2002), $170-183$.

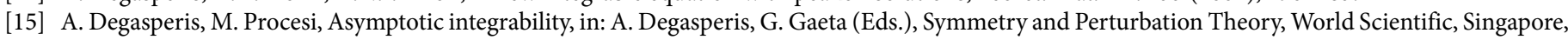
1999, pp. 23-37.

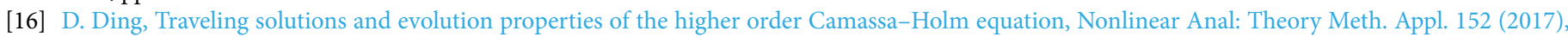
$1-11$.

[17] D. Ding, P. Lv, Conservative solutions for higher-order Camassa-Holm equations, J. Math. Phys. 51 (2010), 072701.

[18] J. Escher, Z. Yin, Well-posedness, blow-up phenomena, and global solutions for the b-equation, J. Reine Angew. Math. 624 (2008), 51-80.

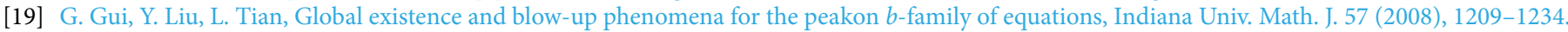

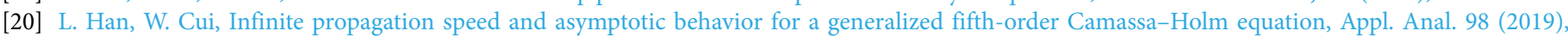
$536-552$.

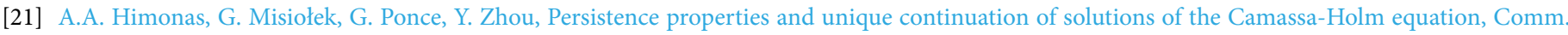
Math. Phys. 271 (2007), 511-522.

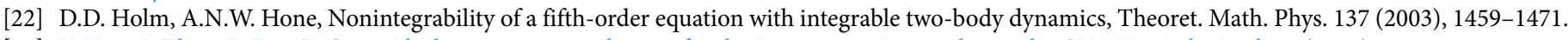

[23] Y. Hou, P. Zhao, E. Fan, Z. Qiao, Algebro-geometric solutions for the Degasperis-Procesi hierarchy, SIAM J. Math. Anal. 45 (2013), $1216-1266$.

[24] Z. Jiang, L. Ni, Y. Zhou, Wave breaking of the Camassa-Holm equation, J. Nonlinear Sci. 22 (2012), $235-245$.

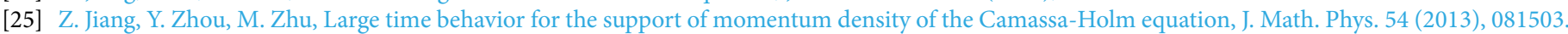

[26] J. Lenells, Traveling wave solutions of the Degasperis-Procesi equation, J. Math. Anal. Appl. 306 (2005), 72-82.

[27] J. Lenells, Conservation laws of the Camassa-Holm equation, J. Phys. A: Math. Gen. 38 (2005), 869-880.

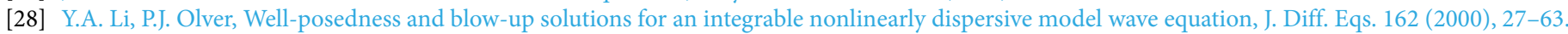

[29] Q. Liu, Z. Qiao, Fifth order Camassa-Holm model with pseudo-peakons and multi-peakons, Int. J. Non-Linear Mech. 105 (2018), $179-185$.

[30] Y. Liu, Z. Yin, Global existence and blow-up phenomena for the Degasperis-Procesi equation, Comm. Math. Phys. 267 (2006), 801-820.

[31] R. McLachlan, X. Zhang, Well-posedness of modified Camassa-Holm equations, J. Diff. Eqs. 246 (2009), $3241-3259$.

[32] H.P. McKean, Breakdown of a shallow water equation, Asian J. Math. 2 (1998), 867-874.

[33] O.G. Mustafa, A note on the Degasperis-Procesi equation, J. Nonlinear Math. Phys. 12 (2005), 10-14.

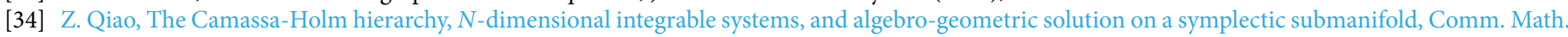
Phys. 239 (2003), 309-341.

[35] Z. Qiao, Integrable hierarchy, 3 × 3 constrained systems, and parametric solutions, Acta Appl. Math. 83 (2004), 199-220.

[36] Z. Qiao, S. Li, A new integrable hierarchy, parametric solutions, and traveling wave solutions, Math. Phys. Anal. Geom. 7 (2004), 289-308.

[37] E.G. Reyes, Geometric integrability of the Camassa-Holm equation, Lett. Math. Phys. 59 (2002), $117-131$.

[38] H. Tang, Z. Liu, Well-posedness of the modified Camassa-Holm equation in Besov spaces, Z. Angew. Math. Phys. 66 (2015), $1559-1580$.

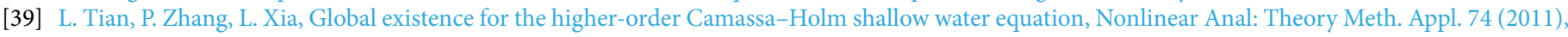
$2468-2474$.

[40] F. Wang, F. Li, Z. Qiao, Well-posedness and peakons for a higher-order $\mu$-Camassa-Holm equation, Nonlinear Anal. 175 (2018), $210-236$.

[41] F. Wang, F. Li, Z. Qiao, On the Cauchy problem for a higher-order $\mu$-Camassa-Holm equation, Discrete Contin. Dyn. Syst. 38 (2018), $4163-4187$.

[42] Y. Zhou, Blow-up phenomenon for the integrable Degasperis-Procesi equation, Phys. Lett. A 328 (2004), 157-162.

[43] Y. Zhou, On solutions to the Holm Staley b-family of equations, Nonlinearity 23 (2010), 369-381. 